\title{
Civilisations
}

Revue internationale d'anthropologie et de sciences

humaines

40-1 | 1991

L'Asie et le Pacifique

\section{La place du Japon}

\section{Yoshiya Kato}

URL : http://journals.openedition.org/civilisations/1684

DOI : 10.4000/civilisations. 1684

ISSN : 2032-0442

\section{Éditeur}

Institut de sociologie de l'Université Libre de Bruxelles

\section{Édition imprimée}

Date de publication : 1 janvier 1992

Pagination : 173-188

ISBN : 2-87263-063-5

ISSN : 0009-8140

\section{Référence électronique}

Yoshiya Kato, « La place du Japon », Civilisations [En ligne], 40-1 | 1991, mis en ligne le 07 juillet 2009, consulté le 30 avril 2019. URL : http://journals.openedition.org/civilisations/1684 ; DOI : 10.4000/ civilisations. 1684

\section{(C) Tous droits réservés}




\section{LA PLACE DU JAPON}

\section{Yoshiya KATO}

1. D'abord quelques données géographiques et géopolitiques :

L'Archipel nippon consistait autrefois en trois grands arcs au large du Continent euro-asiatique et face à l'Océan pacifique. Au nord, les Kouriles, reliant le Kamtchatka à Hokkaido, qui étaient historiquement des territoires japonais. Suite à la seconde guerre mondiale toutefois, nous avons renoncé à la souveraineté sur ces territoires qui maintenant sont reconnus de manière générale comme étant des territoires russes. Quatre îles au sud de cette chaine ne font toutefois pas partie des Kouriles mais de Hokkaido, géologiquement et historiquement. Ces îles sont encore soumises à l'occupation de l'Union Soviétique, et nous ne pourrons jamais accepter cet état de choses car, à part le fait géologique et historique, les Russes eux-mêmes les ont reconnues japonaises par des accords internationaux successifs signés en 1856 et 1875 . Voilà le fameux contentieux territorial entre nous et les Soviétiques, sur lequel je reviendrai plus tard.

$\mathrm{Au}$ centre, se trouvent les quatre grandes îles qui forment le coeur même de l'archipel : Hokkaido, Honshu, Shikoku, Kyushu.

Enfin, au sud, les Ryukyu dont l'île principale est Okinawa, placée sous l'administration américaine après la guerre et restituée au Japon en 1972.

Ce qui est frappant est sans doute le caractère insulaire et l'isolement de notre pays. On compare souvent notre archipel aux Iles britanniques; il est vrai que l'Angleterre et le Japon se 
trouvent chacun à l'extrémité de la grande masse de terre que l'on nomme l'Europe et l'Asie. Mais la proximité avec le grand continent n'est pas la même : la distance entre Calais et Douvres n'est que de $40 \mathrm{~km}$., tandis que celle entre Shimonoseki et Pusan (à la pointe la plus au sud de la péninsule coréenne) est de $200 \mathrm{~km}$., et celle entre Nagasaki et Shanghai $800 \mathrm{~km}$. Le pays le plus proche de nos côtes est, paradoxalement, l'Union Soviétique, même en faisant abstraction des territoires litigieux.

Cet éloignement comporte des avantages et des inconvénients : l'avantage, c'est de rendre l'invasion étrangère très difficile et l'inconvénient, c'est que les communications et les voyages sont parfois très périlleux.

C'est ainsi que l'Archipel nippon constitue une digue contre le vaste océan qui pendant longtemps défiait toute aventure humaine, ainsi qu'un terminus où aboutissent toutes les grandes civilisations d'Orient et d'Occident, où elles s'arrêtent, se figent ou se transforment.

Avec un climat relativement tempéré, avec des paysages variés mais toujours riants, notre pays est néanmoins constamment en proie aux quatre éléments qui s'y abattent. Terre montagneuse et volcanique, d'où des tremblements de terre presque quotidiens; raz de marée, typhons, inondations, qui se répètent chaque année ainsi que les incendies si fréquents La terre arable n'occupe que moins de $15 \%$ de la superficie totale de notre pays, $70 \%$ constituant des forêts et des montagnes; la presque totalité de la population s'accroche sur les côtes et surtout dans des zones urbaines comme Tokyo-Yokohama, Osaka-Kobe ou Fukuoka-Kitakyushu.

Marco Polo, au 14ème siècle dans ses "Récits sur les merveilles du monde" a décrit notre pays sous le nom de 
"Jipangu" comme étant terre couverte d'or, suscitant ainsi la convoitise des européens de l'époque. C'est à la recherche de cette sorte d'"El Dorado" que sont partis les grands voyageurs du temps de la découverte du monde, tels Christophe Colomb, Magellan, Vasco de Gama. Mais en réalité, le Japon n'a jamais été un pays riche en matières premières ni en ressources naturelles : très peu de charbon et minerais, très peu de métaux précieux, pas du tout de pétrole et même pas assez de nourriture.

A l'heure actuelle, le Japon couvre une superficie de 378.000 $\mathrm{km}^{2}$ et compte une population de 120 millions. On pourrait dire grosso modo que le Japon est à peu près douze fois plus grand que la Belgique.

\section{Aperçu historique}

Sans retracer en détail l'histoire de notre pays qui remonte à 2.000 ans, je voudrais relever quatre étapes que nous avons traversées et qui nous ont marquées.

La première est celle de notre contact avec la Chine, foyer d'une grande civilisation, qui date de façon plus ou moins organisée du 6ème siècle après $\mathrm{J}-\mathrm{C}$. De nombreuses délégations, composées non seulement de hauts fonctionnaires mais aussi d'étudiants, de moines novices et d'artisans, ont été envoyées en Chine, sous la Dynastie des Sui et ensuite des T'ang. Ce qu'elles ont apporté de la Chine était bien entendu la culture, les sciences et les techniques chinoises, à l'époque les plus avancées du monde, mais également la religion bouddhiste originaire de l'Inde avec ses soutras, les reliques et divers objets religieux. Pour le Japon resté encore primitif, ce contact avec le continent chinois a donné lieu à des luttes intestines entre anciens et modernes, entre le traditionnel shinto et le nouveau bouddhisme qui, heureusement ont abouti à une sorte 
d'amalgame. Cette époque fut aussi le début de la littérature japonaise, exprimée en japonais mais écrite en caractères chinois d'abord, puis en "kana" (signes phonétiques inventés chez nous), dont un exemple est le "Genji Monogatari" - Le Dit de Genji. Cette rencontre avec la puissance étrangère qu'était la Chine a donné d'autre part naissance à une certaine conscience de l'identité nationale, un éveil d'un certain sentiment patriotique. En 618, le Prince Régent Shotoku a envoyé une lettre à l'Empereur de Chine qui commence par la phrase : "Le Prince du Soleil Levant salue l'Empereur du Soleil Couchant et s'enquiert de sa santé...", ce qui n'avait pas manqué de provoquer la colère de ce dernier.

Le Moyen-Age qui avait ainsi commencé au Japon se termine au 13ème siècle lorsque l'Empire du Mongol Genghiskhan et de ses descendants, après avoir étendu sa domination sur toute la Chine, tenta d'envahir le Japon, et que ces tentatives d'invasion, répétées à deux reprises, ont subi un échec total.

Beaucoup d'entre vous ont sans doute vu à la télévision les funérailles de feu notre Empereur Showa; les costumes des porteurs du palanquin, la musique qui accompagne la marche funèbre, les rites, tout date de cette époque ancienne et moyennageuse, que nous avons conservés jusqu'à nos jours.

La deuxième étape se situe au milieu du 16ème siècle, lorsque nous avons rencontré pour la première fois les Européens. Quelques Portugais naufragés au sud de Kyushu (Tanegashima) en 1542 ont apporté des armes à feu et quelques instruments de navigation. Je voudrais souligner en passant que dix ans après, le Japon était déjà devenu un grand producteur de ces armes à feu, qui ont tellement changé le mode des batailles entre les seigneurs (Daimyo). Les Portugais ont été suivis par les Espagnols, les Anglais et les Hollandais (y compris 
des voyageurs originaires des Pays-Bas méridionaux), et ils ont apporté outre des produits divers d'Occident et d'Orient, les sciences - médicales, physiques, astronomiques, militaires etc.d'Europe, et encore et surtout du christianisme. Les activités de St. François Xavier et des Jésuites se sont répandues dans tout le Japon et le nombre des convertis catholiques s'est accru rapidement - jusqu'à plusieurs centaines de milliers, y compris une dizaine de Daimyo.

Une certaine menace politico-religieuse de la part des puissances catholiques de l'époque fut ressentie de plus en plus fortement par le gouvernement du Shogun, et c'est la raison pour laquelle ce dernier a été amené à la décision de la fermeture du pays à tous les Européens, sauf aux Hollandais protestants.

Le Sakoku (fermeture du pays) a duré pendant deux siècles et le Japon, qui était sur la voie de l'expansion en Asie du SudEst, a été obligé de se replier sur lui-même, de se tenir à l'écart du grand courant mondial. Seul le port de Nagasaki avec le Déjima (promontoire artificel) et une poignée de Hollandais, servait de lucarne ouverte sur le monde occidental.

La troisième vague est celle de la réforme Meiji au milieu du 19 ème siècle, en 1868. Les flottes russe et américaine, ensuite celles des Anglais et des Français, avaient menacé d'ouvrir nos ports pour permettre le commerce avec eux.

Le gouvernement du Shogun Tokugawa, déjà affaibli, ne pouvait plus tenir tête à ces demandes pressantes des puissances européennes; il s'est effondré, et l'autorité impériale a été restaurée au sommet de l'Etat. Sous le règne d'un monarque éclairé, l'Empereur Meiji, le Japon s'est embarqué sur la voie de la modernisation et a réussi tant bien que mal à égaler les autres puissances. 
Les apports des pays européens au Japon moderne sont considérables. Ainsi, l'Angleterre a exercé une influence primordiale dans la mesure où elle s'est liée à notre pays par une alliance militaire, mais les Allemands et les Français ont aussi contribué à la construction de notre état. Les Belges n'ont pas fait exception à cet égard, pour ce qui concerne notamment le système bancaire, les études supérieures en sciences économiques, l'industrie sidérurgique, les chemins de fer, etc.

Il est vrai que les victoires dans les guerres contre la Chine en 1894-95, ensuite contre la Russie tsariste en 1904-05, ont élevé le moral de la nation, mais elles ont également donné naissance à une fâcheuse tendance au militarisme et au nationalisme fanatique. Les militaires se sont de plus en plus accaparés du pouvoir politique, au point de s'arroger même les pouvoirs de l'Empereur. Les aventures militaires contre la Chine, puis dans le Pacifique, contre les Etats-Unis et leurs alliés, la défaite et la capitulation en sont les résultats.

La quatrième et dernière étape est l'époque contemporaine. Le pays était dévasté, l'économie complètement détruite, le moral du peuple au plus bas. Mais c'est alors, si j'ose dire, que la réconciliation s'est réalisée entre les vainqueurs et les vaincus, que le Japon a retrouvé son courage de vivre une vie nouvelle.

Ce sont les Américains qui nous ont aidés, économiquement et politiquement. La fonction impériale a été sauvée, bien que le statut constitutionnel de l'Empereur ait été grandement modifié; une nouvelle constitution, par excellence pacifique, a été promulguée. C'est la période de démocratisation et d'américanisation du pays.

Toutes les données géographiques et historiques que je viens de décrire ont formé la personnalité de la nation japonaise : 
peuple homogène à la mentalité souvent insulaire, timide et fier à la fois, réservé et discipliné d'habitude mais prompt à s'emporter le cas échéant, avide de connaissances mais peu porté à l'épanchement... Elles ont également dicté nos politiques vis-à-vis du monde extérieur, que ce soit dans le domaine économique, culturel ou de défense.

\section{Politique extérieure}

Il existe chez nous une conscience très nette d'appartenance au monde dit "occidental". Cet adjectif "occidental" prête souvent à confusion : il s'agit d'un qualificatif nullement géographique, mais éminemment politique désignant les démocraties libérales. Vu de l'Europe, le Japon est situé à l'est, au-delà de l'Union Soviétique, au-delà de la Chine, bref en un "Extrême-Orient" très éloigné et très différent. Je vous propose donc de regarder vers l'Ouest, au-delà de l'Atlantique, au-delà des Etats-Unis, au-delà même du Pacifique; c'est là que vous trouverez le Japon, c'est-à-dire l'"Extrême Occident" ! Plaisanterie mise à part, nous avons le sentiment très clair que nous partageons avec l'Europe occidentale, les Etats-Unis et le Canada, les mêmes valeurs, le même idéal, un système politique, social et économique identique, basé sur la liberté de pensée et d'expression, le pluralisme et l'alternance. Depuis bientôt un siècle et demi, nous avons appris - d'abord des pays européens, puis des Etats-Unis d'Amérique, la démocratie parlementaire qui est maintenant solidement ancrée au Japon. Nous croyons que nous sommes tous dans le même bateau, ce qui n'est pas nécessairement le cas en Europe.

C'est la raison pour laquelle nous attachons une importance primordiale, dans notre diplomatie, à la solidarité avec les 
autres nations démocratiques et libérales, vouées au même idéal politique.

Les relations avec les Etats-Unis constituent, pour la politique extérieure du Japon, la clef de voûte. Elles sont importantes pour nous à double titre : sécurité et économie. Nos deux pays sont liés par un pacte bilatéral de sécurité par lequel le Japon bénéficie de la dissuasion et de l'aide militaire américaines en cas de guere. Sur le plan économique, nos échanges avec les Etats-Unis se sont développés constamment, malgré les frictions parfois très sérieuses pouvant prendre des allures de guerre commerciale. A l'heure actuelle, le P.N.B. des Etats-Unis et celui du Japon additionnés représentent un tiers du P.N.B. mondial, et nos relations sont, comme le disait l'ex-Ambassadeur Mike Mansfield, les relations bilatérales les plus importantes dans le monde.

Viennent ensuite nos rapports avec la Communauté Européenne et les autres pays de l'Europe de l'Ouest. A la différence de nos relations avec les Etats-Unis, nous n'avons de lien de sécurité et de défense avec aucun des pays européens; nous ne sommes pas, non plus, membre de l'OTAN. Dans le domaine économique également, nos relations ont souvent été jusqu'à présent plutôt concurrentielles que coopératives. En vue de la construction européenne et du marché unique de 1992, nous sommes maintenant convaincus de la nécessité de renforcer nos liens économiques et politiques avec l'Europe. En fin de compte, une Europe unifiée et plus forte serait dans notre intérêt et dans l'intérêt du monde entier.

L'Europe, le Japon et les Etats-Unis constituent les trois piliers principaux du monde actuel et il est de notre devoir à tous les trois de maintenir et de développer les relations triangulaires de manière équilibrée et harmonieuse. 
Voilà le premier volet de notre politique étrangère. Le deuxième volet concerne nos relations avec les pays d'Asie et du Pacifique. Ethniquement et culturellement, nous autres Japonais sommes asiatiques, cela est indéniable. Mais l'Asie est si vaste, si variée et si complexe; la plupart des pays de cette région sont encore en voie de développement.

Il faudrait signaler d'autre part que ces derniers temps certains pays et régions comme la Corée du Sud, Taiwan, Singapour et Hong Kong ont connu un développement remarquable, et ces dragons de l'Asie, avec les pays de l'ASEAN (les Philippines, la Thailande, la Malaisie, l'Indonésie notamment) constituent une nouvelle force qui fait son apparition sur l'échiquier mondial.

Il faudrait se rappeler dans ce contexte d'une thèse philosophique, prophétique aussi, d'après laquelle le 21ème siècle serait le siècle de l'Asie-Pacifique et que le centre de gravité de la civilisation, passant de la Méditerranée à l'Atlantique, est maintenant en train de se déplacer vers le Pacifique.

Le Japon, une puissance économique et un élément de stabilité dans cette région, est certainement appelé à jouer un rôle toujours croissant pour le développement économique et social de celle-ci ainsi que pour sa stabilisation politique.

Au printemps 1988, M. Takeshita, alors Premier ministre, a défini la politique étrangère qu'il entendait poursuivre et qu'il appellait "concept de la coopération internationale" en trois points :

1. coopération pour la paix

2. aide officielle au développement et

3. échanges culturels internationaux. 
Le premier point désigne entre autres la coopération et la participation pour les opérations de maintien de la paix mises en oeuvre par les Nations-Unies; la deuxième est l'augmentation de nos aides économiques et techniques aux pays en voie de développement; et le troisième, peut-être le plus intéressant pour vous Européens, comprend non seulement le programme d'échange d'étudiants et de professeurs, mais également les manifestations culturelles, telles que les expositions Europalia-Japon par exemple.

4. Le problème de défense pour le Japon contemporain a ceci de particulier quaux termes de la Constitution de 1946, nous avons renoncé à la guerre comme droit souverain de la nation et que nous ne nous reconnaissons plus le droit de belligérance. Il en découle que nos forces armées ne sont pas une armée à proprement parler, que les opérations militaires sont strictement limitées pour la défense de notre territoire et que nos troupes ne peuvent pas être envoyées hors de nos frontières... Cette Constitution fut rédigée dans l'immédiat après-guerre et sous une forte influence américaine. Nous adhérons aussi à ce que nous appelons "les trois principes nonnucléaires", à savoir ne pas détenir, ne pas fabriquer et ne pas laisser entrer des armes nucléaires sur notre sol. A l'intérieur de ces limitations constitutionnelles et des restrictions volontaires, nous nous sommes efforcés de mettre au point nos capacités de défense; à l'heure actuelle, nos forces de défense comptent 230.000 hommes au total, 255.000 tonnes de bâtiments de guerre, 350 avions militaires - des forces donc à peu près comparables à celles de l'Italie; par exemple sur le plan budgétaire, le Japon se situe en 9ème position, après la République Fédérale d'Allemagne et avant l'Italie, avec 12 milliards de dollars, soit approximativement $1 \%$ du P.N.B.

Tout comme les membres européens de l'OTAN, nous faisons face au problème du "burden sharing" dans le cadre du 
Traité de sécurité nippo-américain, et tout comme eux, nous sommes confrontés à la nouvelle diplomatie de paix lancée par M. Gorbatchev.

Mais la situation stratégique et politique est très différente en Asie et en Europe. En Europe règne la confrontation des deux blocs : l'OTAN et le Pacte de Varsovie, bien que des négociations de désarmement soient menées entre eux, tandis qu'en Asie, la situation militaire est beaucoup plus complexe, marquée par la présence de l'URSS, de la Chine, des Etats-Unis, de la Corée divisée, de Taiwan séparé du continent chinois etc. et la démarcation entre les deux camps ne peut pas être tracée. Quant à nous, ce qui rend notre position plus difficile encore vis-à-vis de l'Union Soviétique, c'est l'existence du problème territorial non résolu, en raison duquel nous n'avons même pas conclu de traité de paix avec l'URSS. Vous vous souvenez sans doute que l'Allemagne fédérale sous le Chancelier Brandt avait lancé, il y a un quart de siècle, la fameuse Ost-Politik qui, en reconnaissant les frontières actuelles et la division de l'Allemagne en deux, avait abouti à une série d'accords entre la R.F.A. et les pays de l'Est, et frayé le chemin pour les négociations Est-Ouest. Une situation comparable n'existe pas encore dans notre voisinage et la "perestroika" et la diplomatie de la nouvelle pensée qui fascinent les pays occidentaux, surtout au niveau de l'opinion publique, ne se font pas sentir encore, au moins en ce qui concerne le Japon.

Pourtant, je voudrais souligner que, malgré ces différences stratégiques et politiques entre l'Europe et l'Asie, la paix et la sécurité sont indivisibles et qu'il faut aborder ces problèmes de sécurité de façon globale. Ceci est particulièrement vrai de nos jours où le globe terrestre est devenu si petit et que les moyens de transports et les techniques militaires sont si avancées. L'accord sur les missiles nucléaires à portée moyenne (INF) a 
pleinement démontré que la menace des armes nucléaires est partout pareille, que ce soit en Europe ou en Asie. La solution régionale du problème de désarmement n'est pas une vraie solution, le désarmement en Europe seule n'est pas le vrai désarmement. Ce principe d'indivisibilité s'applique aux armes conventionnelles comme les chars et les canons, aussi bien qu'aux missiles. C'est pourquoi nous suivons de près et avec beaucoup d'intérêt les négociations sur les armes conventionnelles qui se déroulent à Vienne.

5. Enfin, l'économie. Le Japon représente à lui seul $10 \%$ du P.N.B. mondial à l'heure actuelle. Ajoutez à cela le dynamisme, l'organisation et la discipline à la japonaise, qui permettent d'appeler notre pays - et ceci sans orgueil ni prétention - une puissance économique. Nous sommes conscients des responsabilités qui en découlent aussi.

Sur le plan de la politique économique, nos soucis majeurs sont, en premier lieu, de maintenir et développer nos relations avec les Etats-Unis, le plus important partenaire commercial pour nous, de façon à éviter dans la mesure du possible, les frictions inévitables. Nos exportations et importations avec les Etats-Unis sont de plus de 130 milliards de dollars, occupant $29 \%$ de nos échanges globaux. Les relations américano-japonaises sont très importantes, non seulement pour l'économie de chacun de nos deux pays, mais aussi pour la stabilité politique et la prospérité de la région Asie-Pacifique tout entière.

En deuxième lieu, nous sommes quelque peu préoccupés par l'horizon 1992 de la Communauté Européenne. Le volume de nos échanges avec la CEE est de 71 milliards de dollars, soit $15,7 \%$ de l'ensemble de nos exportations et importations. Il est bien évident que la construction européenne est souhaitable pour un meilleur équilibre mondial, et que le marché unique 
européen avec 320 millions d'habitants constituera un élément positif pour l'économie mondiale. Nous sommes convaincus aussi que l'Europe ne sera pas, ne pourrait être, une forteresse, mais devrait être ouverte au monde extérieur, comme vous le répétez sans cesse. Il reste à savoir, pourtant, quelle sera la modalité d'uniformisation et quelle sera la condition de l'ouverture de vos marchés aux partenaires non-européens. La fameuse thèse de la "réciprocité" demeure encore peu claire pour nous, et nous craignons que lorsqu'on forme une flotte ou un convoi de navires, l'on ajuste la vitesse de l'ensemble à celle du bateau le plus lent.

Je voudrais signaler en troisième lieu les progrès remarquables des pays de l'Asie du Sud, notamment des "dragons", qui font l'objet non moins important de nos politiques économiques. Le triangle formé par le Japon, les Etats-Unis et le Sud-Est Asiatique constitue maintenant une région aux activités économiques très intenses, avec des possibilités futures d'expansion très grandes.

Le problème du Sud - pauvreté, famine, sousdéveloppement, dettes accumulées - attire également toute notre attention. C'est la raison pour laquelle $M$. Takeshita, dans sa conception de la coopération internationale en trois points que je viens de mentionner, met l'accent sur l'accroissement de nos aides officielles au tiers monde.

Le Japon, pays insulaire et isolé, nation commerçante plus que celle des Samourai, a besoin du commerce extérieur, a besoin de la paix mondiale, et ce peut-être plus que n'importe quel autre pays. Nous sommes les adeptes probablement les plus zélés du principe du libre-échange, et pour ne pas nous trahir à cet égard, nous avons déployé tous nos efforts pour éliminer les obstacles et entraves d'ordre commercial qui existaient et protégeaient auparavant notre marché intérieur. 
L'époque a évolué du nationalisme économique vers le globalisme des échanges, de l'autarcie et du régionalisme vers l'interdépendance et l'inter-développement. A la fin de ce siècle, somme toute si mouvementé et si dramatique, nous devrions comprendre, Européens, Américains et Japonais tous pareillement, cette réalité des choses, à savoir le rapetissement de la terre et la nécessité de vivre ensemble en coopérant. Voilà le meilleur gage pour le 21ème siècle qui nous attend. 


\section{SUMMARY}

A rapid description of Japan's geographical position provides an explanation for her territorial disputes and her traditional isolation. Japan's history is summarised in four stages, each of which brought her into contact with new countries and contributed to the formation of her national identity.

Japan's present international relations are characterised by the fact that she belongs to the Western world, by the importance for her of the United States and Western Europe, and by the part which she is called upon to play in the AsiaPacific region. The particular conditions which determine her thinking in matters of security explain the limits placed upon the development of her defensive capacity and her interest in the progress of international negotiations on disarmament.

Japan is aware of the responsibilities imposed upon her by her economic power with respect to the stability and prosperity of the Asia-Pacific region. To the essential economic relations that she maintains with the United States, the countries of the EEC and the newly industrialised economies along the Asian rim of the Pacific must be added her cooperation with the countries of the Third World in recognition of the real interdependence which unites the nations. 
